\title{
STABILIZATION OF SOLUTIONS OF AN EXTERIOR BOUNDARY VALUE PROBLEM FOR SOME CLASS OF EVOLUTION SYSTEMS
}

\author{
B. V. KAPITONOV
}

Institute of Mathematics, Russian Academy of Sciences, Siberian Branch

Universitetskiu Prosp. 4, 630090 Novosibirsk, Russia

Let $\Omega \subset \mathbb{R}^{n}$ be the complement of the closure of a bounded domain which is starlike with respect to the origin and has smooth boundary $S$. Let $\mathcal{L}\left(x, \mathcal{D}_{x}\right)$ be a strongly elliptic operator of order $2 l(l \geq 1)$ in $\Omega$. In $\Omega \times(0, T)$ we consider the initial boundary value problem

$$
\begin{gathered}
u_{t t}+\mathcal{L}\left(x, \mathcal{D}_{x}\right) u=0, \\
\left.u\right|_{t=0}=f_{1}(x),\left.\quad u_{t}\right|_{t=0}=f_{2}(x), \\
\left.\mathcal{B}_{j}\left(x, \mathcal{D}_{x}, \mathcal{D}_{t}\right) u\right|_{S}=0, \quad j=1, \ldots, l,
\end{gathered}
$$

where the boundary operators $\mathcal{B}_{j}$ are such that problem (1) is well posed.

Our purpose is to study asymptotics as $t \rightarrow \infty$ of solutions of this problem for some class of operators $\mathcal{L}$ and $\mathcal{B}_{j}$.

Many authors have studied the behavior as $t \rightarrow \infty$ of solutions of exterior boundary value problems for hyperbolic equations. These questions have been most completely investigated for the wave equation: qualified decay has been obtained (powerlike in the case of an even number of space variables and exponential in the case of an odd number). The work in this direction can be divided into two groups, each with its own approach.

The first approach consists in application of the Laplace transform in the variable $t$ with subsequent study of the analytic properties of the resolvent and its behaviour for the large and small values of the spectral parameter. In [14], [19], [20] this program is realized for various boundary problems for the wave equation $\left(-\mathcal{L}\left(x, \mathcal{D}_{x}\right)=\Delta_{n}\right.$, the Laplace operator in $\left.x_{1}, \ldots, x_{n} ; n=2,3\right)$ in the exterior of a bounded convex domain.

The second approach, based on nonstandard energy identities, was applied by C. Morawetz to the wave equation with the Dirichlet boundary condition 
in the exterior of a starlike domain. In a number of papers (see [1], [22]-[24]) the method of integral identities was applied to the case of a moving obstactle (the boundary $S$ depends on $t$ ) and also to a hyperbolic equation with variable coefficients whose leading part coincides with the wave operator outside some ball. Within this approach, in [2]-[7], [13], [18], [21] qualified decay was obtained for the first boundary value problem for the wave equation in an exterior domain for which the starlike property was replaced by a more general one, for the Maxwell system with the Leontovich boundary condition and for the linear system of elasticity theory with the Dirichlet condition on the boundary.

Nonstandard energy identities for the wave equation were obtained by C. Morawetz in [15], [16] by the $a, b, c$-method, and in [17] by means of the Kelvin transform.

In this paper, in studying the behaviour of solutions of problem (1) we use a modification of the second approach. Our method applies not only to hyperbolic equations (systems) but also to equations describing perturbations propagating with infinite speed.

In the cylinder $\Omega \times(0, T)$ we consider the initial-boundary value problem

$$
\begin{gathered}
\frac{\partial^{2} u}{\partial t^{2}}+(-1)^{l} \sum_{i, j} \frac{\partial^{l}}{\partial x_{i_{1}} \ldots \partial x_{i_{l}}}\left(A^{i, j}(x) \frac{\partial^{l} u}{\partial x_{i_{1}} \ldots \partial x_{i_{l}}}\right)=0 \\
\left.u\right|_{t=0}=f_{1}(x),\left.\quad \frac{\partial u}{\partial t}\right|_{t=0}=f_{2}(x), \\
\left.\mathcal{D}_{x}^{\alpha} u\right|_{S}=0, \quad|\alpha|=\left|\left(\alpha_{1}, \ldots, \alpha_{n}\right)\right| \leq l-1,
\end{gathered}
$$

where $i=\left(i_{1}, \ldots, i_{l}\right), j=\left(j_{1}, \ldots, j_{l}\right), i_{p}, j_{q} \in\{1, \ldots, n\}, u=\left(u^{1}(x, t), \ldots\right.$ $\left.\ldots, u^{m}(x, t)\right), A^{i, j}(x)$ are $m \times m$ matrices, $A^{i, j}(x)=\left(A^{j, i}(x)\right)^{*}, A^{i, j}(x)=A_{0}^{i, j}+$ $A_{1}^{i, j}(x), A_{1}^{i, j}(x)=o(1)$ as $|x| \rightarrow \infty$.

We assume that there exists a constant $c>0$ such that

$$
\sum_{i, j} A^{i, j}(x) \xi_{j} \bar{\xi}_{i} \geq c \sum_{i}\left|\xi_{i}\right|^{2}, \quad x \in \Omega,
$$

where $\xi_{j}=\left(\xi_{j}^{1}, \ldots, \xi_{j}^{m}\right)$ are arbitrary complex-valued vectors.

We introduce the following notations:

$$
\begin{gathered}
A u=(-1)^{l} \sum_{i, j} \frac{\partial^{l}}{\partial x_{i_{1}} \ldots \partial x_{i_{l}}}\left(A^{i, j}(x) \frac{\partial^{l} u}{\partial x_{j_{1}} \ldots \partial x_{j_{l}}}\right), \\
2 \Phi(u)=\sum_{i, j} A^{i, j}(x) \frac{\partial^{l} u}{\partial x_{j_{1}} \ldots \partial x_{j_{l}}} \cdot \frac{\partial^{l} \bar{u}}{\partial x_{i_{1}} \ldots \partial x_{i_{l}}}, \\
J_{0}(u(x, t))=\left|\frac{\partial u}{\partial t}\right|^{2}+2 \Phi(u), \quad J_{k}(u(x, t))=J_{0}\left(\frac{\partial^{k} u}{\partial t^{k}}\right), \quad k \geq 1 .
\end{gathered}
$$

Assume that $f_{k} \in C^{\infty}(\Omega), k=1,2 ; f_{2}(x) \equiv 0$ for $|x| \geq R_{0},|x|^{p} \mathcal{D}_{x}^{\beta} f_{1} \in L_{2}(\Omega)$ for all $p \geq 0,|\beta| \geq 0 ;\left.\mathcal{D}_{x}^{\alpha}\left(A^{s} f_{k}\right)\right|_{S}=0$ for $|\alpha| \leq l-1, s=0,1, \ldots$ 
We define the local energy of order $k$ of a solution $u(x, t)$ of problem $(2)$ at time $t$ concentrated in the region $\Omega_{R}=\{x \in \Omega|| x \mid<R\}$ by

$$
E_{R}^{k}(t)=\int_{\Omega_{R}} J_{k}(u(x, t)) d x
$$

The following notion is very useful for the purpose of our work. We call the quantity

$$
E^{k, \varrho}(t)=\int_{\Omega} \frac{1}{|x|^{\varrho}} J_{k}(u(x, t)) d x \quad\left(\varrho \in \mathbb{R}^{1}\right)
$$

the weighted energy of order $k$ of the solution $u(x, t)$ of problem (2).

It is a well-known result that the behaviour as $t \rightarrow \infty$ of the local energy depends on $n$ (the number of space variables) and $l$ (the order of the operator $A$ ).

Our first result shows that the behaviour as $t \rightarrow \infty$ of the weighted energy depends neither on $n$ nor on $l$. Here we make the following assumption on $A^{i, j}(x)$ :

$$
\sum_{i, j}\left(\frac{\partial}{\partial|x|} A^{i, j}(x)\right) \xi_{j} \cdot \bar{\xi}_{i} \leq 0 \quad \text { for } x \in \Omega .
$$

TheOREM 1. Let $u(x, t)$ be a solution of problem (2) and let $q \in \mathbb{N}$. Suppose that the above assumptions on $f_{1}, f_{2}, A^{i, j}(x)$ are satisfied and

$$
A^{i, j}(x) \in C^{l+q}(\Omega), \quad\left|\mathcal{D}_{x}^{\beta} A^{i, j}(x)\right| \leq \frac{C}{|x|^{|\beta|+q}} \quad \text { for } 1 \leq|\beta| \leq l+q .
$$

Then there exist constants $C_{1}, C_{2}>0$ such that for $k=0,1, \ldots, q$,

$$
\frac{C_{1}}{t^{\varrho}} \leq \int_{\Omega} \frac{1}{|x|^{\varrho}} J_{k}(u(x, t)) d x \leq \frac{C_{2}}{t^{\varrho}}, \quad t \geq 1,-\infty<\varrho \leq k .
$$

COROLlaRY. The local energy of order $k$ of a solution $u(x, t)$ of problem (2) satisfies the estimate

$$
\int_{\Omega_{R}} J_{k}(u(x, t)) d x \leq \frac{C_{2}}{t^{k}}, \quad t \geq 1, k=0,1, \ldots, q .
$$

R e mark. If the $A^{i, j}(x)$ satisfy some additional condition the inequalities of Theorem 1 hold for $-\infty<\varrho \leq k+\varkappa, k \geq 1,0<\varkappa \leq 1$.

We now consider the initial-boundary value problem with $t$-periodic right side:

$$
\begin{gathered}
v_{t t}+A v=e^{i \omega t} g(x), \\
\left.v\right|_{t=0}=f_{1}(x),\left.\quad v_{t}\right|_{t=0}=f_{2}(x),\left.\quad \mathcal{D}_{x}^{\alpha} v\right|_{S}=0, \quad|\alpha| \leq l-1,
\end{gathered}
$$

where $\omega \in \mathbb{R}^{1}, g(x) \equiv 0$ for $|x| \geq R_{0}, g \in C^{\infty}(\Omega),\left.\mathcal{D}_{x}^{\alpha}\left(A^{s} g\right)\right|_{S}=0$ for $|\alpha| \leq l-1$, $s=0,1, \ldots$.

Using Theorem 1 we study the behaviour as $t \rightarrow \infty$ of the solutions of problem (3).

We begin with the principle of limiting absorption. 
Let $z$ be a complex number and $\operatorname{Im} z<0$. We denote by $W(x, z)$ a solution in $H^{2 l}(\Omega)$ of the boundary value problem

$$
A W+(i z)^{2} W=g,\left.\quad \mathcal{D}_{x}^{\alpha} W\right|_{S}=0, \quad|\alpha| \leq l-1 .
$$

It is not hard to show that this problem has a unique solution in $H^{2 l}(\Omega)$. On the other hand, problem (4) has no solution in $H^{2 l}(\Omega)$ for $z$ such that $\operatorname{Im} z=0$. In this case (4) has many solutions in $H_{|x|^{-3}}^{2 l}(\Omega)$, a space with the norm

$$
\|u\|=\left(\sum_{|\beta| \leq 2 l}\left\||x|^{-3 / 2} \mathcal{D}_{x}^{\beta} u\right\|_{L_{2}(\Omega)}^{2}\right)^{1 / 2} .
$$

Theorem 2 (The principle of limiting absorption). Suppose $\omega \neq 0, \operatorname{Im} \omega=0$, $A^{i, j}(x) \in C^{l+3}(\Omega)$, and

$$
\left|\mathcal{D}_{x}^{\beta} A^{i, j}(x)\right| \leq \frac{C}{|x|^{|\beta|+3}} \quad \text { for } 1 \leq|\beta| \leq l+3 .
$$

Then the limit

$$
\lim _{z \rightarrow \infty, \operatorname{Im} z<0} W(x, z)=w(x, \omega) \quad \text { exists in } H_{|x|^{-3}}^{2 l}(\Omega) .
$$

Moreover, $w(x, \omega)$ is a solution of problem (4) for $z=\omega$ and

$$
\int_{\Omega} \frac{1}{|x|^{3}}\left|\mathcal{D}_{x}^{\beta}(W(x, z)-w(x, \omega))\right|^{2} d x \leq C(\alpha, \beta)|z-\omega|^{\alpha}, \quad 0<\alpha<1,|\beta| \leq 2 l .
$$

This solution $w(x, \omega)$ plays an important role in the following assertion:

TheOREM 3 (The principle of limiting amplitude). Let $v(x, t)$ be a solution of problem (3), $\omega \neq 0, q \in \mathbb{N}, q \geq 3$. Suppose $A^{i, j}(x) \in C^{l+q}(\Omega)$ and

$$
\left|\mathcal{D}_{x}^{\beta} A^{i, j}(x)\right| \leq \frac{C}{|x|^{|\beta|+q}} \quad \text { for } 1 \leq|\beta| \leq l+q .
$$

Then for $k=3, \ldots, q$,

$$
\int_{\Omega} \frac{1}{|x|^{k}} J_{k}\left(v(x, t)-e^{i \omega t} w(x, \omega)\right) d x \leq \frac{C(\delta)}{t^{k-2-\delta}}, \quad 0<\delta<1,
$$

where $w(x, \omega)$ is the function constructed in Theorem 2 .

Remark. If $\omega=0$, then the solution $v(x, t)$ of problem (3) satisfies the estimate

$$
\int_{\Omega} \frac{1}{|x| \varrho} J_{k}(v(x, t)) d x \leq \frac{C_{2}}{t^{\varrho}}, \quad \varrho \leq k-1,1 \leq k \leq q .
$$

Remark. For a generalization of the system (1):

$$
u_{t t}+\sum_{l=1}^{p}(-1)^{l} \sum_{i, j} \frac{\partial^{l}}{\partial x_{i_{1}} \ldots \partial x_{i_{l}}}\left(A^{i, j}(x) \frac{\partial^{l} u}{\partial x_{j_{1}} \ldots \partial x_{j_{l}}}\right)+Q(x) u=0,
$$

our results are valid under some assumptions on $A^{i, j}(x)$. 
We note that the inequalities of Theorem 1 are sharp estimates of the weighted energy. We are now going to improve the estimates of the local energy for some special operators $A$.

In the cylinder $\Omega \times(0, T)$ we consider the initial boundary value problem

$$
\begin{gathered}
\varrho u_{t t}=\mu \Delta_{n} u+(\lambda+\mu) \operatorname{grad}(\operatorname{div} u), \\
\left.u\right|_{t=0}=\varphi(x),\left.\quad u_{t}\right|_{t=0}=\psi(x),\left.\quad u\right|_{S}=0,
\end{gathered}
$$

where $u=\left(u^{1}, \ldots, u^{n}\right), u^{i}=u^{i}(x, t)$, and $\varrho, l, m$ are positive constants.

For $n=3$, the system in (5) is the classical system of equations for elastic waves. In this case $u$ is the displacement vector, $\varrho$ is the density of the medium, and $\lambda$ and $\mu$ are the Lamé constants.

We assume that the supports of the initial data lie in a ball of radius $a$.

THEOREM 4. For any $R>$ a there exists a constant $C(a, R)>0$ such that a solution $u(x, t)$ of problem (5) satisfies the inequality

$$
\int_{\Omega_{R}} J_{0}(u(x, t)) d x \leq \frac{C(a, R)}{t} \int_{\Omega} J_{0}(u(x, 0)) d x, \quad t \geq 1 .
$$

Moreover, there exists a constant $C_{k}(\varphi, \psi, R)>0$ such that for $t \geq 2$

$$
\int_{\Omega_{R}} J_{k}(u(x, t)) d x \leq C_{k}(\varphi, \psi, R) \frac{\ln t}{t^{k+1}}, \quad k=1,2, \ldots
$$

Remark. The first estimate of Theorem 4 has been obtained by G. Das$\operatorname{sios}[3]$.

In the case of $n$ odd, the exponential decay of a solution of problem (5) can be established from (6) and the Huygens principle.

TheOREM 5. Let $\mathcal{D} \subset \Omega$ be an arbitrary domain lying in a ball of radius $d$. There exist positive constants $\beta(a)$ and $C(a, d)$ such that

$$
\int_{D} J_{0}(u(x, t)) d x \leq C e^{-\beta t} \int_{\Omega} J_{0}(u(x, 0)) d x, \quad t>(a+d) \sqrt{\varrho / \mu} .
$$

Applying these results we can improve the estimates of Theorem 3 for system (5) with $t$-periodic right side.

Consider now the Maxwell system. In $\Omega \times(0, T)(n=3)$ we consider the initial-boundary value problem for the Maxwell system

$$
\begin{gathered}
\left\{\begin{array}{l}
e_{t}=\operatorname{curl}(\mu h), \\
h_{t}=-\operatorname{curl}(\lambda e), \\
\operatorname{div} e=\operatorname{div} h=0,
\end{array}\right. \\
\left.e\right|_{t=0}=e^{0}(x),\left.\quad h\right|_{t=0}=h^{0}(x), \quad[v, e]-\left.\alpha(h-v(h, v))\right|_{S}=0,
\end{gathered}
$$

where $e$ and $h$ are three-dimensional vector-valued functions of $t, x=\left(x_{1}, x_{2}, x_{3}\right)$, $v$ is the unit outer normal, $[\cdot, \cdot]$ and $(\cdot, \cdot)$ are the vector and inner products, 
$\mu=\mu(x)$ and $\lambda=\lambda(x)$ are scalar functions in $\Omega$ and $\alpha=\alpha(x)$ is a continuously differentiable nonzero function on $S$ with $\operatorname{Re} \alpha \geq 0$.

Let $\lambda(x)$ and $\mu(x)$ be continuously differentiable functions in $\bar{\Omega}$ satisfying the conditions

$$
\begin{gathered}
C_{1} \leq \lambda(x) \leq C_{2}, \quad C_{1} \leq \mu(x) \leq C_{2}, \\
C_{1}>0, \quad|\nabla \lambda|,|\nabla \mu| \leq C_{3} .
\end{gathered}
$$

We denote by $\mathcal{H}$ the Hilbert space of pairs $\left\{u_{1}, u_{2}\right\}$ of three-component complexvalued functions $u_{k} \in L_{2}(\Omega)$ with the inner product

$$
\left\langle\left\{u_{1}, u_{2}\right\},\left\{v_{1}, v_{2}\right\}\right\rangle_{0}=\int_{\Omega}\left(\lambda\left(u_{1}, \bar{v}_{1}\right)+\mu\left(u_{2}, \bar{v}_{2}\right)\right) d x .
$$

We denote by $\mathcal{H}_{1}$ the Hilbert space consisting of pairs $u=\left\{u_{1}, u_{2}\right\}$ such that $\left\{u_{1}, u_{2}\right\},\left\{\operatorname{curl} u_{1}, \operatorname{curl} u_{2}\right\} \in \mathcal{H}$. We define the inner product in $\mathcal{H}_{1}$ by

$$
\begin{array}{r}
\langle u, v\rangle_{1}=\int_{\Omega}\left[\left(\operatorname{curl} \lambda u_{1}, \operatorname{curl} \lambda \bar{v}_{1}\right)+\left(\operatorname{curl} \mu u_{2}, \operatorname{curl} \mu \bar{v}_{2}\right)\right. \\
+(c+\lambda)\left(u_{1}, \bar{v}_{1}\right) \\
\left.+(c+\mu)\left(u_{2}, \bar{v}_{2}\right)\right] d x,
\end{array}
$$

where the constant $c$ is chosen so that the norm in $\mathcal{H}_{1}$ is equivalent to the norm defined by the expression

$$
\left(\int_{\Omega}\left[\left|\operatorname{curl} u_{1}\right|^{2}+\left|\operatorname{curl} u_{2}\right|^{2}+\left|u_{1}\right|^{2}+\left|u_{2}\right|^{2}\right] d x\right)^{1 / 2} .
$$

Lemma. Suppose $\alpha \in C^{1}(S)$. The mapping $u \rightarrow\left[v, u_{1}\right]-\alpha\left(u_{2}-v\left(u_{2}, v\right)\right)$ from $C_{\mathrm{c}}^{1}\left(\bar{\Omega}=\left\{u \in C^{1}(\bar{\Omega})\right.\right.$ with compact support $\}$ into $C^{1}(S)$ extends by continuity to a continuous linear mapping of $\mathcal{H}_{1} \rightarrow H_{-1 / 2}(S)$ which we also denote by $u \rightarrow\left[v, u_{1}\right]-\alpha\left(u_{2}-v\left(u_{2}, v\right)\right) \equiv \varkappa(\alpha, u)$.

The lemma makes it possible to introduce in $\mathcal{H}_{1}$ the closed subspace $\stackrel{\circ}{\mathcal{H}}_{1}(\alpha)=$ $\left\{u \in \mathcal{H}_{1}: \varkappa(\alpha, u)=0\right\}$, which is dense in $\mathcal{H}$.

In $\mathcal{H}$ we define the unbounded operator $A$ by $\mathcal{D}(A)=\stackrel{\circ}{\mathcal{H}}_{1}(\alpha), A u=\left\{\operatorname{curl} \mu u_{2}\right.$, $\left.-\operatorname{curl} \lambda u_{1}\right\}$ for $u \in \mathcal{D}(A)$.

It can be shown that $\mathcal{D}\left(A^{*}\right)=\stackrel{\circ}{\mathcal{H}}_{1}(-\bar{\alpha})$ and

$$
A^{*} v=-\left\{\operatorname{curl} \mu u_{2},-\operatorname{curl} \lambda u_{1}\right\} \quad \text { for } v=\left\{v_{1}, v_{2}\right\} \in \mathcal{D}\left(A^{*}\right) .
$$

Let $M=\left\{v \in \mathcal{D}\left(A^{*}\right): A^{*} v=0\right\}$, and let $M_{1}$ be the orthogonal complement of $M$ in $\mathcal{H}$. We remark that elements $\left\{u_{1}, u_{2}\right\} \in M_{1} \cap \mathcal{D}(A)$ have the following property: $\operatorname{div} u_{i}=0$ in the sense of distributions.

We assume that $f=\left\{e^{0}, h^{0}\right\} \in M_{1} \cap \mathcal{D}\left(A^{3}\right)$. It is not hard to show that in this case the solution $u=\{e, h\}$ belongs to the space $H_{2}(\Omega)$, while curl $e$, curl $h$, $\operatorname{curl}^{2} e, \operatorname{curl}^{2} h \in \mathcal{H}_{1}(\Omega)$. 
We introduce the notations:

$$
\begin{aligned}
& E_{0}(\{e, h\}(t), R)=\int_{\Omega_{R}}\left(\lambda|e|^{2}+\mu|h|^{2}\right) d x, \\
& E_{n}(\{e, h\}(t), R)=E_{0}\left(A^{n}\{e, h\}(t), R\right),
\end{aligned}
$$

where $\{e, h\}$ is a solution of problem $(7)$, and

$$
I(t, R)=E_{0}(\{e, h\}(t), R)+E_{2}(\{e, h\}(t), R) .
$$

The main result of this part of the paper can be stated as follows:

THEOREM 6. Suppose $\{e, h\}$ is a solution of problem (7), the initial data $f=$ $\left\{e^{0}, h^{0}\right\}$ belongs to $M_{1} \cap \mathcal{D}\left(A^{3}\right), f(x) \equiv 0$ for $|x| \geq a, \lambda, \mu \in C^{3}(\bar{\Omega}), \partial \lambda / \partial|x|$, $\partial \mu / \partial|x| \leq 0, \lambda(x) \equiv \mu(x) \equiv 1$ for $|x| \geq b, \alpha \in C^{1}(S), \operatorname{Re} \alpha \geq 0$, and

$$
\min \left\{\lambda|\alpha|^{2}, \mu\right\}-3 \operatorname{Re} \alpha|\tan (v, x)| \sqrt{\lambda \mu}>0 .
$$

Then for any $r>0$ there are constants $\beta=\beta(a)$ and $C=C(a, r)>0$ such that

$$
I(t, r) \leq C \exp (-\beta t) I(0, a) .
$$

We finish by studying the problem of diffraction of electromagnetic waves.

Let $B_{m}$ be a bounded 3-dimensional domain, starlike with respect to the origin, with smooth boundary $S_{m}$. We assume that $B_{m} \subset B_{m+1}$ for $m=1, \ldots, k-1$. We set $\Omega_{m}=B_{m+1} \backslash \bar{B}_{m}$ for $m=1, \ldots, k-1, \Omega_{k}=\mathbb{R}^{3} \backslash \bar{B}_{k}, \Omega_{0}=B_{1}, v$ is the unit outer normal on $S_{m}$.

We consider the system

$$
\begin{array}{cl}
\frac{\partial E}{\partial T}=\operatorname{curl}(\mu(x) H), & \frac{\partial H}{\partial t}=-\operatorname{curl}(\lambda(x) E), \\
\left.E\right|_{t=0}=f_{1}(x), & \left.H\right|_{t=0}=f_{2}(x) .
\end{array}
$$

Suppose that $\lambda, \mu$ are smooth functions in $\Omega_{m}$ and have discontinuities on $S_{m}$. The conditions on $S_{m}$ then have the form

$$
\begin{array}{ll}
{\left.\left[v, \lambda^{m} E^{m}\right]\right|_{S_{m}}=\left.\left[v, \lambda^{m-1} E^{m-1}\right]\right|_{S_{m}},} & m=1, \ldots, k, \\
{\left.\left[v, \lambda^{m} H^{m}\right]\right|_{S_{m}}=\left.\left[v, \lambda^{m-1} H^{m-1}\right]\right|_{S_{m}},} & m=1, \ldots, k,
\end{array}
$$

where $\lambda^{m}, \mu^{m}, E^{m}, H^{m}$ are the restrictions of the corresponding functions on $\Omega_{m}$.

We would like to study the behaviour as $t \rightarrow \infty$ of the local energy of solution of problem (8)-(9):

$$
E_{R}(t)=\int_{|x|<R}\left(\lambda|E|^{2}+\mu|H|^{2}\right) d x
$$

TheOREM 7. Suppose the initial data $f=\left\{f_{1}, f_{2}\right\}$ belongs to $\mathcal{D}(A) \cap M_{1}$ (where $A$ is defined in a similar way to Theorem 6$)$ and $f(x) \equiv 0$ for $|x| \geq b$. Assume that the functions $\lambda$ and $\mu$ satisfy the following conditions:

$$
\lambda^{j}(x)<\lambda^{j-1}(x), \quad \mu^{j}(x)<\mu^{j-1}(x), \quad x \in S_{j}, j=1, \ldots, k,
$$




$$
\begin{gathered}
\frac{\partial \lambda^{j}}{\partial|x|} \leq 0, \quad \frac{\partial \mu^{j}}{\partial|x|} \leq 0, \quad x \in \Omega_{j}, j=0,1, \ldots, k, \\
\lambda(x) \equiv \mu(x) \equiv 1 \quad \text { for }|x| \geq b .
\end{gathered}
$$

Then for any $r>0$ there are constants $\beta=\beta(b)>0$ and $C=C(b, r)>0$ such that

$$
E_{r}(t) \leq C \exp (-\beta t) E_{b}(0) .
$$

Rem ark. The behaviour as $t \rightarrow \infty$ of solutions of the diffraction problem for acoustic waves has been investigated in [11].

The proofs of Theorems 1-7 can be found in [4]-[11].

\section{References}

[1] C. Bloom, A rate of approach to the steady state of solutions of second-order hyperbolic equation, J. Differential Equations 19 (1975), 296-329.

[2] C. Bloom and H. Kazarinoff, Local energy decay for a class of nonstarshaped bodies, Arch. Rational Mech. Anal. 55 (1974), 73-85.

[3] G. Dassios, Local energy decay for scattering of elastic waves, J. Differential Equations 49 (1983), 124-141.

[4] B. V. Kapitonov, On the behavior, as $t \rightarrow \infty$, of solutions of an exterior boundary value problem with the Leontovich condition for the Maxwell system, Soviet Math. Dokl. 37 (1988), 377-380.

[5] -, On exponential decay as $t \rightarrow \infty$ of solutions of an exterior boundary value problem for the Maxwell system, Math. USSR-Sb. 66 (1990), 475-498.

[6] - On the decay as $t \rightarrow \infty$ of the solutions of the Cauchy problem for the Maxwell system in an inhomogeneous medium, in: Qualitative Analysis of Solutions of Partial Differential Equations, S. K. Godunov (ed.), Inst. Mat., Sibirsk. Otdel. Akad. Nauk SSSR, Novosibirsk 1985, 100-109 (in Russian).

[7] -, On the decay of the solution of an exterior boundary value problem for the linear system of elasticity, Differentsial'nye Uravneniya 22 (1986), 452-458 (in Russian).

[8] -, Decay of solutions of an exterior boundary value problem and the principle of limiting amplitude for a hyperbolic system, Soviet Math. Dokl. 33 (1986), 243-247.

[9] -, Behavior as $t \rightarrow \infty$ of the solutions of the exterior boundary-value problem for a hyperbolic system, Siberian Math. J. (1988), 444-457.

[10] - On the decay as $t \rightarrow \infty$ of solutions of an exterior boundary value problem for a strongly hyperbolic system, Soviet Math. Dokl. 41 (1990), 236-240.

[11] - Stabilization of solutions and the principle of limiting absorption for a high order system, preprint 23, Inst. Mat., Sibirsk. Otdel. Akad. Nauk SSSR, Novosibirsk 1989 (in Russian).

[12] —, Stabilization of solutions of mixed problems for a class of evolution systems, doctoral thesis, Novosibirsk 1990 (in Russian).

[13] G. P. Menzala, Large time behavior of elastic waves in inhomogeneous medium, Boll. Un. Mat. Ital. B (7) 3 (1985), 95-108.

[14] V. P. Mikhailov, On the principle of limiting amplitude, Dokl. Akad. Nauk SSSR 159 (1964), 750-752 (in Russian).

[15] C. Morawetz, The decay of solutions of the exterior initial-boundary value problem for the wave equation, Comm. Pure Appl. Math. 14 (1961), 561-568.

[16] —, The limiting amplitude principle, ibid. 15 (1962), 349-361. 
[17] C. Morawetz, Energy decay for star-shaped obstacles, Appendix 3 to: P. D. Lax and R. S. Phillips, Scattering Theory, Academic Press, 1967, 261-264.

[18] C. Morawetz, J. Ralston and W. Strauss, Decay of solutions of the wave equation outside nontrapping obstacles, Comm. Pure Appl. Math. 30 (1977), 447-508.

[19] L. A. Murave $\breve{1}$, Asymptotic behaviour for large values of time of solutions of the second and third exterior boundary value problems for the wave equation with two space variables, Trudy Mat. Inst. Steklov. 126 (1973), 73-144 (in Russian).

[20] - On the asymptotic behaviour for large values of time of a solution of an exterior boundary value problem for the wave equation, Dokl. Akad. Nauk SSSR 220 (1975), 289292 (in Russian).

[21] W. Strauss, Dispersal of waves vanishing on the boundary of an exterior domain, Comm. Pure Appl. Math. 28 (1975), 265-278.

[22] H. Tamura, On the decay of the local energy for wave equations with a moving obstacle, Nagoya Math. J. 71 (1978), 125-147.

[23] —, Local energy decays for wave equations with time-dependent coefficients, ibid. 107-123.

[24] E. C. Zachmanoglou, The decay of solutions of the initial-boundary value problem for hyperbolic equations, J. Math. Anal. Appl. 13 (1966), 504-515. 